\title{
Fractional power series method for solving fractional differemtial
}

\section{equation}

\author{
Runqing Cui and Yue $\mathrm{Hu}$
}

School of Mathematics and Informatics, Henan Polytechnic University, Jiaozuo 454003, China Igdcuirunqing@yeah.net, huu3y6@163.com

\section{Abstruct:}

we use fractional power series method (FPSM) to solve some linear or nonlinear fractional differential equations . Compared to the other method, the FPSM is more simple, derect and effective.

Keyword: fractional power series: fractional differential equation: Caputo fractional derivative.

\section{Introduction}

Many phenomena in applied sciences and engineering can be decribed successfully by using fractional order differential equation [1-7]. But fractional order differential equations are usually hard to solve analytically and exact solution are rather difficult to be obtained. So it is very important to find efficient methods for solving these equations.

Various researchers have introduced new methods in the literature. These methods include variational iteration method (VIM) [1], homotopy perturbation method (HPM) [2], homotopy analysis method (HAM) [3], DGJ method [5], Adomian decomposition method (ADM) [4], and other methods [7].

In this paper, we will use fractional power series method (FPSM) [10] to solve some fractional differential equations. Compared to the above method, the FPSM is more simple and effective.

\section{Basic definitions}

Definition 1.([6] ) A power series representation of the form

$$
\sum_{n=0}^{\infty} c_{n}\left(t-t_{0}\right)^{n \alpha}=c_{0}+c_{1}\left(t-t_{0}\right)^{\alpha}+c_{2}\left(t-t_{0}\right)^{2 \alpha}+\cdots
$$

where $0 \leq m-1<\alpha \leq m, m \in N^{+}$and $t \geq t_{0}$ is called a fractional power series (FPS) about $t_{0}$, where $t$ is a variable and $c_{n}$ are the coeffients of the series.

Definition 2. ( [7] ) The fractional derivative of $f(x)$ in Caputo sense is defined as

$$
D^{\alpha} f(x)=\frac{1}{\Gamma(m-\alpha)} \int_{0}^{x}(x-s)^{m-\alpha-1} f^{(m)}(s) d s,
$$

for $m-1<\alpha \leq m, m \in N^{+}, x>0$.

I n addition, we also need the following property: 
Theorem 1. ([6]) Suppose that the FPS $\sum_{n=0}^{\infty} c_{n} t^{n \alpha}$ has radius of convergence $R>0$. If $f(t)$ is a function defined by $f(t)=\sum_{n=0}^{\infty} c_{n} t^{n \alpha}$ on $0 \leq t<R$, then for $m-1<\alpha \leq m$ and $0 \leq t<R$, we have:

$$
D^{\alpha} f(t)=\sum_{n=0}^{\infty} c_{n} \frac{\Gamma(n \alpha+1)}{\Gamma((n-1) \alpha+1)} t^{(n-1) \alpha}
$$

\section{Illustrative examples}

In this section, the applicability of FPSM shall be demonstrated by two test examples.

\subsection{Example 1}

First, we consider the fractional telegraph equation in the form [8]:

$$
D_{x}^{\alpha} u=\frac{\partial^{2} u}{\partial t^{2}}+\frac{\partial u}{\partial t}+u
$$

with the initial conditions

$$
u(0, t)=e^{-t}, u_{x}^{\prime}(0, t)=0 .
$$

Where $1<\alpha \leq 2$ is a parameter describing the order of the fractional space derivative. To apply FPSM , suppose that the solution of (4) takes the form:

$$
u(x, t)=\sum_{k=0}^{\infty} a_{k}(t) x^{\alpha k}=a_{0}(t)+a_{1}(t) x^{\alpha}+a_{2}(t) x^{2 \alpha}+\cdots
$$

Using (5), we have $a_{0}(t)=e^{-t}$. Next we determine the $a_{k}(t)(k=1,2, \cdots)$.

From Theorem 1, we obtain

$$
D_{x}^{\alpha} u(x, t)=\sum_{k=1}^{\infty} \frac{a_{k}(t) \Gamma(\alpha k+1)}{\Gamma(\alpha(k-1)+1)} x^{\alpha(k-1)} .
$$

On the other hand,

$$
\begin{gathered}
\frac{\partial^{2} u}{\partial t^{2}}=\frac{\partial^{2} a_{0}(t)}{\partial t^{2}}+\frac{\partial^{2} a_{1}(t)}{\partial t^{2}} x^{\alpha}+\frac{\partial^{2} a_{2}(t)}{\partial t^{2}} x^{2 \alpha}+\cdots \\
\frac{\partial u}{\partial t}=\frac{\partial a_{0}(t)}{\partial t}+\frac{\partial a_{1}(t)}{\partial t} x^{\alpha}+\frac{\partial a_{2}(t)}{\partial t} x^{2 \alpha}+\cdots
\end{gathered}
$$

Substituting (6), (7), (8) and (9) into (4), and comparing the coefficients of $x^{\alpha}$ in the both side, we get 


$$
a_{k}(t)=\frac{e^{-t}}{\Gamma(\alpha k+1)}, \quad(k=1,2, \cdots)
$$

Thus we obtain the solution

$$
u(x, t)=\sum_{k=0}^{\infty} \frac{e^{-t}}{\Gamma(\alpha k+1)} x^{\alpha k} .
$$

The exact solution to (4) when $\alpha=2$ is given as:

$$
u(x, t)=e^{-t} \cos h x
$$

\subsection{Example 2}

Now, we consider the fractional Riccati equation that is frequently encountered in optimal control problem [7]:

$$
D^{\beta} u(t)=2 u(t)-u^{2}(t)+1,0<\beta<1, \quad 0<t<1,
$$

with the initial condition $u(0)=0$.

To apply FPSM , we suppose that the solution of (4) takes the form:

$$
u(t)=a_{0}+a_{1} t^{\beta}+a_{2} t^{2 \beta}+a_{3} t^{3 \beta}+\cdots
$$

Using the initial condition $u(0)=0$, we have $a_{0}=0$. Next we determine the $a_{k}(k=1,2, \cdots)$.

By Theorem 1, we get

$$
D^{\beta} u=a_{1} \frac{\Gamma(\beta+1)}{\Gamma(1)}+a_{2} \frac{\Gamma(2 \beta+1)}{\Gamma(\beta+1)} t^{\beta}+a_{3} \frac{\Gamma(3 \beta+1)}{\Gamma(2 \beta+1)} t^{2 \beta}+\cdots
$$

Substitute (11) and (12) into (10), yields that:

$$
\begin{gathered}
a_{1} \frac{\Gamma(\beta+1)}{\Gamma(1)}+a_{2} \frac{\Gamma(2 \beta+1)}{\Gamma(\beta+1)} t^{\beta}+a_{3} \frac{\Gamma(3 \beta+1)}{\Gamma(2 \beta+1)} t^{2 \beta}+\cdots \\
=2 \sum_{k=1}^{\infty} a_{k} t^{\beta k}-\left(\sum_{k=1}^{\infty} a_{k} t^{\beta k}\right)^{2}+1 .
\end{gathered}
$$

The equating of the coefficients of $t^{\beta k}$ in both side of (13), leads to the following:

$$
a_{1}=\frac{1}{\Gamma(\beta+1)},
$$




$$
\begin{gathered}
a_{2}=\frac{2}{\Gamma(2 \beta+1)}, \\
a_{3}=\frac{4 \Gamma^{2}(1+\beta)-\Gamma(1+2 \beta)}{\Gamma^{2}(1+\beta) \Gamma(1+3 \beta)},
\end{gathered}
$$

and so on.

Thus the solution can be obtained. For example, when $\beta=0.5$, we have the solution as

$$
u=\frac{2}{\sqrt{\pi}} t^{\frac{1}{2}}+2 t+\frac{16(\pi-1)}{3 \pi^{3 / 2}} t^{\frac{3}{2}}-\frac{\pi-4}{\pi} t^{2}+\cdots
$$

which agrees with the results of this problem obtained in [7]

\section{Conclusion}

we have used FPSM to solve some linear or nonlinear fractional differential equations . Compared to the other method, the FPSM is more simple, derect and effective.

\section{References}

1. He $\mathrm{J} \mathrm{H}$. Variational iteration method-a kind of non-linear analytical technique: some examples[J]. International journal of non-linear mechanics, 1999, 34(4): 699-708.

2. He J H. Homotopy perturbation method: a new nonlinear analytical technique[J]. Applied Mathematics and computation, 2003, 135(1): 73-79.

3. Liao S. Beyond perturbation: introduction to the homotopy analysis method[M]. CRC press, 2003.

4. Adomian G. A review of the decomposition method in applied mathematics[J]. Journal of mathematical analysis and applications, 1988, 135(2): 501-544.

5. Sari M, Gunay A, Gurarslan G. A solution to the telegraph equation by using DGJ method[J]. International Journal of Nonlinear Science, 2014, 17(1): 57-66.

6. El-Ajou A, Arqub O A, Zhour Z A, et al. New results on fractional power series: theories and applications[J]. Entropy, 2013, 15(12): 5305-5323.

7. Elsaid A. Fractional differential transform method combined with the Adomian polynomials[J]. Applied Mathematics and Computation, 2012, 218(12): 6899-6911.

8. Cascaval R C, Eckstein E C, Frota C L, et al. Fractional telegraph equations[J]. Journal of Mathematical Analysis and Applications, 2002, 276(1): 145-159. 